\title{
Application of GEANT4 radiation transport toolkit to dose calculations in anthropomorphic phantoms
}

\author{
P. Rodrigues ${ }^{1}$, A. Trindade ${ }^{1}$, L. Peralta $^{1,2}$, \\ C. Alves $^{3}$, A. Chaves ${ }^{3}$, M.C. Lopes ${ }^{3}$
}

In this paper we present the implementation of a dose calculation application, based on the GEANT4 Monte Carlo toolkit. Validation studies were performed with an homogeneous water phantom and an Alderson-Rando anthropomorphic phantom both irradiated with high-energy photon beams produced by a clinical linear accelerator. As input, this tool requires computer tomography images for automatic codification of voxel based geometries and phase space distributions to characterize the incident radiation field. Simulation results were compared with ionization chamber, thermoluminescent dosimetry data and commercial treatment planning system calculations. In homogeneous water phantom, overall agreement with measurements were within 1-2\%. For anthropomorphic simulated setups (thorax and head irradiation) mean differences between GEANT4 and TLD measurements were less than $2 \%$. Significant differences between GEANT4 and a semi-analytical algorithm implemented in the treatment planning system, were found in low density regions, such as air cavities with strong electronic disequilibrium.

\footnotetext{
${ }^{1}$ LIP - Laboratório de Instrumentação e Física Experimental de Partículas, Portugal

${ }^{2}$ FCUL - Faculdade de Ciências da Universidade de Lisboa, Portugal

${ }^{3}$ IPOFG/CROC - Instituto Português de Oncologia Francisco Gentil, Centro Regional de Oncologia de Coimbra, Portugal
} 


\section{Introduction}

The need to predict dose is a common requirement of several research fields such as external and internal radiotherapy $[1,2]$, nuclear medicine imaging [3], radionuclide therapy [4], hadrontherapy [5], radioprotection in high-energy accelerators, commercial air flights [6] and space dosimetry [7].

For dose calculations deterministic or semi-analytical tools have been developed, which relied in approximations to Boltzmann transport equation. Most of these methods are inherently not exact and do not account for secondary particle production, but are still used, specially in radiotherapy, where a fast high-volume tool tailored to clinical demands is required $[8,9]$. The probability of treatment success or tumor control probability in radiotherapy strongly depends on delivering an adequately high dose to the intended target volume. At the same time, the radiation tolerance of the normal tissue near the tumor is regarded as a limiting factor. Particularly for some tumors and normal tissues, a small change in dose, can result in a large change in probability of tumor control and normal tissue secondary effects. This leads to stringent limits on accuracy in dose calculation which are currently set at $2-5 \%$ [10]. In order to cope with this requirement, quality control of radiotherapy treatment planning systems must be performed. For this purpose several quality control protocols have been developed, such as the Report 55 by AAPM Task Group 23 for external photon beams [11].

Knowledge on the limitations presented by semi-analytical algorithms have favored the introduction of Monte Carlo (MC) techniques in dose calculations, since they allow a detailed treatment of the physics processes involved in the interaction of ionizing radiation with matter. Several advances in the Monte Carlo modeling of radiation transport key-areas, such as new electron and photon transport mechanics, improved physics models and significant decrease in computational time allowed a wide-spread of this technique.

GEANT4 is an object-oriented Monte Carlo simulation toolkit[12], developed by the RD44 (up to 1998) and GEANT4 Collaboration, which provides a new approach for development of accurate dose calculations applications. GEANT4 includes an extended set of electromagnetic and hadronic physics models across a wide energy range. The same physics process can be treated by alternative implementations which can have different energy validity ranges, accuracy and computational time. In addition, the user can add new physics models in GEANT4 without the need to modify the existing code. Geometry modeling capability is also of paramount importance for realistic and efficient dose calculations. In this area, GEANT4 provides a large set of solids of different complexity and allows an efficient repetitive structure representation.

This paper presents the implementation of a dose calculation application based on the GEANT4 toolkit and is organized as follows: in the first section a brief overview of a GEANT4 based dose calculation application is given, with emphasis on CT image interface and basic validation procedures. In the following sections, dose measurements and comparison with GEANT4 calculations for different Alderson-Random irradiation configurations are described. 


\section{GEANT4-based dose calculation application}

\subsection{System organization}

The main components of the developed dose calculation application are: the GEANT4 kernel, which performs event-by-event transport in a voxelized userdefined geometry (section 2.2); a CT image data interface and correspondent material parameterisation; a primary event generator interface to phase-space distributions (section 2.3), which describe the incident radiation field and a data analysis service, to account for dose deposition in the geometry (Figure ??). Data analysis was accomplished by means of the ROOT [13] data analysis framework, although an abundant number of alternative data analysis AIDA-compliant [14] solutions are also available in GEANT4. Simulations were performed on a PC-farm running Linux Red Hat 6.1 and a PBS scheduler for job submission.

\subsection{Geometry implementation}

Our GEANT4-based application was designed in order to perform dose calculations in 3D voxel rectilinear geometries. Accurate representation of the human body is achieved with the aid of radiological images, namely those from Computerized Axial Tomography (CT) [15]. CT multi-slice data is acquired along the plane perpendicular to axial axis (from feet to head) or trans-axial plane. In most exams, CT images slices are usually spaced by $3-5 \mathrm{~mm}$ and organized in a $512 \times 512$ pixel matrix. Each pixel is coded with a given CT number $(\mathrm{H})$, which in Hounsfield units is defined as:

$$
H=\left(\frac{\bar{\mu}}{\bar{\mu}_{H_{2} O}}-1\right) \times 1000
$$

were $\bar{\mu}$ and $\bar{\mu}_{H_{2} O}$ are mean values of X-ray photon linear attenuation coefficient for a given material and water, respectively. The pixel size is a function of the scanner Field-Of-View (FOV), ranging from $0.65 \mathrm{~mm}$ to $1 \mathrm{~mm}$. The use of multi-slice CT data requires however a large number of volumes (several millions). To avoid inherent high memory requirements, GEANT4 toolkit offers the concept of parameterised placements [12]. This type of specialized physical volumes are used to represent repetitive structures, so that multiple copies of the same volume can be assigned with different materials. In this representation, the material is parameterised as a function of the voxel localization. During program initialization $\mathrm{CT}$ images are read and information on the allocated material to each voxel is established. These images were previously converted from native DICOM standard to ASCII format. The correspondence between each voxel CT number and its material is established by calibration functions, which are CT scanner dependent. For the used scanner, a Siemens Somatom Plus 4, CT number conversion to elemental weights of tissue and mass density were obtained following the work of Schneider et al [16]. These authors have divided the CT number range, comprehended between -1000 Hounsfield to 1500 Hounsfield, in different regions, each one having a distinct density and elemental weights of tissue parameterization. For CT numbers comprehended 
between 100 Hounsfield $\leq H \leq 1524$ Hounsfield, mass density corresponding to bone region is given by:

$$
\rho(H)=\left(1.017+0.592 \times 10^{-3} H\right) \mathrm{g} \mathrm{cm}^{-3}
$$

Soft tissues are divided into two distinct regions -98 Hounsfield $\leq H \leq 14$ Hounsfield and 23 Hounsfield $\leq H \leq 100$ Hounsfield yielding:

$$
\begin{aligned}
& \rho(H)=\left(1.018+0.893 \times 10^{-3} H\right) \mathrm{g} \mathrm{cm}^{-3} \\
& \rho(H)=\left(1.003+1.169 \times 10^{-3} H\right) \mathrm{g} \mathrm{cm}^{-3}
\end{aligned}
$$

while for the lung region -1000 Hounsfield $\leq H \leq-98$ Hounsfield:

$$
\rho(H)=\left(1.21 \times 10^{-3}+(H-1000) \frac{-0.93+1.21 \times 10^{-3}}{+98-1000}\right) \mathrm{g} \mathrm{cm}^{-3}
$$

For the CT number conversion to elemental weights, the scale of Hounsfield units was divided into 24 bins. The first bin $(-1000$ Hounsfield $\leq \mathrm{H} \leq-950$ Hounsfield) is assigned to the composition of air and the second one $(-950 \leq \mathrm{H} \leq$ $-120)$ with the composition of lung. For the other bins, elemental weights were obtained from interpolation functions to selected tissues (red/yellow marrow, skeleton cortical bone, adipose tissue, adrenal gland, small intestine wall and connective tissue). CT number to mass density parameterisation is displayed in Figure 2. This parameterisation is used to identify the voxel material during particle transport. In each voxel, dose was accounted and information on the physical interaction of a track in a given material was recorded, after each transport step.

\subsection{Incident radiation field}

The simulation of each event in the phantom starts after it is provided the relevant information regarding one or more primary particles, characterized by their kinematics. For this purpose we have interfaced GEANT4 with precalculated phase-space data obtained by MC simulations of a $6 \mathrm{MV}$ photon beam produced by a Siemens Mevatron KD2 linear accelerator [17]. The phasespace data is a small subset of the particles present in the radiation field and includes variables like energy, position, direction and charge for each particle (in this case photons, electrons and positrons). For the developed application phase-space data describes the radiation field just below the Siemens Mevatron KD2 secondary collimators and is transported downstream in the subsequent GEANT4 calculations. In alternative to phase-space distributions, the user can also provide analytical beam modeling functions.

\subsection{GEANT4 physics list}

GEANT4 allows the user to just choose the relevant physics processes for a specific application. In radiotherapy dose calculations with photon beams up to $\approx 7 \mathrm{MeV}$ both Low Energy Electromagnetic Process [18] for photons (photoelectric effect, pair production, Compton and Rayleigh scattering, transport 
down to $250 \mathrm{eV}$ ) and Standard Process for electrons and positrons (multiple Coulomb scattering, bremsstrahlung emission, ionization and $\delta$-ray production, transport down to $10 \mathrm{keV}$ ) were considered. No hadronic interactions were registered in the Physics List, since at these energies photo-nuclear process have a small cross section. However, for dose calculations with higher energy photon beams up to $25 \mathrm{MeV}$, near the giant dipole resonance region, GEANT4 provides a description of photo-nuclear process that can be used to determine the dose contribution that arises from the reaction products like heavy recoils and neutrons.

\subsection{Basic system validation}

\section{Dose calculations}

The simulation of the interaction of a $6 \mathrm{MV}$ photon beam, produced by a Siemens Mevatron KD2 accelerator, with a $50 \times 50 \times 50 \mathrm{~cm}^{3}$ water phantom was performed for different field sizes. The simulated central axis depth dose and dose profile distributions for $10 \times 10 \mathrm{~cm}^{2}$ - Figure 3 - and $15 \times 15 \mathrm{~cm}^{2}-$ Figure 4 - field sizes were compared with measured dose curves obtained with a PTW-233642 ${ }^{1}, 0.125 \mathrm{~cm}^{3}$ sensitive volume ionization chamber. Differences $(\Delta)$ between GEANT4 dose values (normalized to the maximum dose) $\left(D_{G E A N T 4}\right)$ and experimental dose values (normalized to its maximum dose) $\left(D_{D a t a}\right)$ were calculated as:

$$
\text { Difference }=D_{G E A N T 4}(\%)-D_{\text {Data }}(\%)
$$

Deviations up to $4 \%$ due to averaging effects both from histogram binning and ionization chamber size, are seen near the phantom water surface in the buildup region of the depth dose curve. This discrepancy can be removed by a smaller binning $(\approx 1 \mathrm{~mm})$ in Monte Carlo simulations and the use of extrapolation chambers in experimental measurements [19]. Along the central axis for greater depths, an agreement within $2 \%$ was found for both fields - Figure 5 . GEANT4 dose profile distributions show deviations less than $2 \mathrm{~mm}$ when compared with experimental data.

\section{CT interface validation}

Validity of CT numbers calibration was cross-checked for the Alderson-Rando phantom. A CT thorax image was acquired and two regions composed of different radiological substitute tissues were identified, Rando-lung and Randotissue [20], and the CT-number distributions obtained. Mean CT values were then used to calculate a mean density value for this tissue using parameterizations described in [16]. Results were compared with manufacturer data and are presented in Table 1. With this calibration one can predict mass density from $\mathrm{CT}$ data with a accuracy better than $0.02 \mathrm{gcm}^{-3}$. CT number to chemical composition calibration was also assess using Alderson-Rando tissues. Significant differences were found for carbon and oxygen percentage composition. However

\footnotetext{
${ }^{1}$ PTW, Freibug, Germany
} 
their combined weight are very similar to those tabulated in agreement with Schneider et al [20]. For synthetic lung and tissue combined weights for carbon and oxygen were found to be $85.9 \%$ and $86.5 \%$, respectively. These are in fair agreement with tabulated values of $92.1 \%$ (Rando lung) and $84.8 \%$ (Rando tissue).

\section{Irradiation of an Alderson-Rando Phantom}

For our study an Alderson-Rando ${ }^{2}$ anthropomorphic phantom (type AverageMan Rando Phantom), representing a human male body, with $175 \mathrm{~cm}$ height and $73.5 \mathrm{~kg}$ weight was used. The Alderson-Rando phantom is made of soft tissue-equivalent Rando-tissue $\left(\rho=0.98 \pm 0.01 \mathrm{gcm}^{-3}\right)$, lung tissue-equivalent plastic Rando-lung $\left(\rho=0.32 \pm 0.01 \mathrm{gcm}^{-3}\right)$ and includes a human bone skeleton. Each anthropomorphic phantom is transected-horizontally in $2.5 \mathrm{~cm}$ thick slices. Each slice has holes where thermoluminescent dosimeters can be inserted.

Phantom irradiation was performed in a Siemens Mevatron KD2 linear accelerator with $6 \mathrm{MV}$ photon beams for different field sizes at $100 \mathrm{~cm}$ Sourceto-Surface Distance. The following irradiation configurations were used:

1. Alderson-Rando phantom thorax region with a single $15 \times 15 \mathrm{~cm}^{2}$ lateral field

2. Alderson-Rando phantom head region with two $5 \times 5 \mathrm{~cm}^{2}$ opposed lateral fields

3. Alderson-Rando phantom head region with two $10 \times 10 \mathrm{~cm}^{2}$ opposed lateral fields

\subsection{Dose measurements}

For the different irradiation setups experimental data was obtained with thermoluminescent dosimeters (TLD) TLD $-100^{3}$ chips with a $4.5 \mathrm{~mm}$ diameter and $1 \mathrm{~mm}$ thickness. The TLD readout equipment was constituted by the automated PCL3 reader and the annealing oven, model ETT, both from Fimel/PTW. The reference dosimetric system toward which TLDs were calibrated was a thimble ionization chamber, PTW-M31003 ${ }^{4}$, and the associated UNIDOS electrometer. Each TLD sensitivity was taken into account by the assignment of a chip specific correction factor. Calibration procedures were performed for the 6 MV photon beam produced by the Mevatron KD2 linear accelerator and in geometric conditions as close as possible to the ones above established. With this purpose TLD chips were inserted into the allowed positions inside the AldersonRando phantom slices. Experimental uncertainties in dose measurements were estimated in $3-4 \%$.

\footnotetext{
${ }^{2}$ The Phantom Laboratory, New York, USA

${ }^{3}$ Bicron/Saint-Gobain Industrial Ceramics, France

${ }^{4}$ PTW, Freibug, Germany
} 


\subsection{Dose calculations}

Dose calculations for the irradiation of Alderson-Rando phantom were performed with GEANT4 using a phase-space data description for $6 \mathrm{MV}$ photon beams with field dimensions ranging from $5 \times 5$ to $15 \times 15 \mathrm{~cm}^{2}$. Results were compared with a commercial treatment planning system, $\mathrm{PLATO}^{5}$ (version 2.2.23) available at $I P O F G / C R O C$ and currently used in clinical practice. This system uses a semi-analytical bidimensional pencil-beam formalism for photon dose calculations with heterogeneity correction algorithm [21, 22]. PLATO calculates dose to water, disregarding mass stopping power differences between different media and takes only into account density differences by means of dedicated CT number parameterization.

CT images, required for geometry definition in PLATO and GEANT4 systems were acquired with a Siemens Somatom Plus 4 scanner at $120 \mathrm{kVp}$ and then transferred to GEANT4 and PLATO. For thorax and lung irradiation a single CT image with a $51 \mathrm{~cm}$ FOV and $512 \times 512$ voxels of $1 \times 1 \times 5 \mathrm{~mm}^{3}$ (thickness) was used - Figure 6 . In order to reduce the total CPU time required for dose calculations and due to the presence of large homogeneous regions, this image was resized to a new $128 \times 128$ voxels image, with $4 \times 4 \times 5 \mathrm{~mm}^{3}$ voxels. For head region irradiation a total of $37 \mathrm{CT}$ images, with $512 \times 512$ voxels for a $33.4 \mathrm{~cm}$ FOV were acquired and latter resized to $128 \times 128$ voxels with dimensions $2.6 \times 2.6 \times 5 \mathrm{~mm}^{3}$ (thickness) - Figure 7 . In GEANT4 simulations, production cutoffs for secondary particles were set to $1 \mathrm{~mm}$ for photons (250 eV equivalent energy cutoffs in water) and $20 \mu \mathrm{m}$ for electrons and positrons (33.8 keV equivalent energy cutoffs in water). Both GEANT4 and PLATO dose calculation results were stored in matrices with the same resolution of the CT images in order to allow direct data intercomparisons.

\section{Results and Discussion}

\subsection{Anthropomorphic thorax irradiation}

Data analysis for configuration (1) was performed by comparing central axis depth dose and profile distributions at 9.8 and $25 \mathrm{~cm}$ from the beam entrance point. GEANT4 and PLATO dose values were normalized to $D_{0}$, the dose value at $6.5 \mathrm{~cm}$ depth along the beam central axis. For each TLD, differences to calculated relative dose values were assessed according with equation (6). The fit quality between GEANT4 and PLATO to experimental measurements was assessed through the calculation of $\chi^{2}$ and reduced $\chi^{2}$ values.

GEANT4 calculated central axis depth dose - Figure 8 a) - agrees with TLD data, with a maximum deviation of $2.4 \%$. In the region, between $10 \mathrm{~cm}$ and $20 \mathrm{~cm}$, where transitions from lung tissue to soft tissue are observed, PLATO overestimates dose from 6.0 up to $10.7 \%$ (at $15.8 \mathrm{~cm}$ depth). In profile dose distribution at $9.8 \mathrm{~cm}$ depth simulation results show a maximum deviation of $2.6 \%$ from measured data. Similar agreement is observed at $25 \mathrm{~cm}$ depth Figure 8 b) - with maximum differences from experimental data of $1.6 \%$ for

\footnotetext{
${ }^{5}$ Nucletron BV, Holland
} 
GEANT4. Both for $9.8 \mathrm{~cm}$ and $25 \mathrm{~cm}$ profiles, PLATO results show differences up to $5.3 \%$ and $5.6 \%$ respectively. For all 21 dosimeters the differences (TLD - GEANT4) and (TLD - PLATO) are displayed in figure 9. Uncertainties in (TLD - GEANT4) differences are due to quadratic sum of statistical Monte Carlo and TLD errors. For (TLD - PLATO) results, errors take only into account TLD measurement errors. $\chi^{2}$ values - Table 2 - indicate that for this irradiation configuration GEANT4 dose calculations $\left(\chi^{2} / n d f=0.52^{6}\right)$ correctly describe the experimental data. For PLATO a $\chi^{2} / n d f=6.71$ was obtained due to large dose overestimation in lung-soft tissue interfaces. This kind of effects are of clinical relevance in the case of a tumor located near such interfaces. In these scenario PLATO results would induce an underdosage delivered, and a correspondent decrease in tumor control probability [23].

\subsection{Anthropomorphic head irradiation simulation}

In configuration (2) the irradiation of Alderson-Rando phantom head region was performed with two $5 \times 5 \mathrm{~cm}^{2}$ opposed lateral fields. No significant differences were found between GEANT4 $\left(\chi^{2} / n d f=0.83\right)$, PLATO $\left(\chi^{2} / n d f=0.80\right)$ and experimental data - Figure 10. The good agreement seen between PLATO, experimental data and GEANT4 reflects the fact that irradiation with this small field occurs on a region almost without significant heterogeneities - Figure 7 . Maximum deviations obtained with GEANT4 and PLATO were $6.6 \%$ and $8.1 \%$. They were both seen for the same dosimeter, which had in the two irradiation sessions a variability $\left(D_{\text {session } 1}-D_{\text {session } 2}\right) / D_{\text {mean }} \times 100 \%$ of $5.6 \%$.

For configuration (3) $\left(10 \times 10 \mathrm{~cm}^{2}\right.$ opposed lateral fields $) \chi^{2} / n d f=0.93$ and $\chi^{2} / n d f=1.69$ were obtained with GEANT4 - Figure 11 a) - and PLATO Figure 11 b) - respectively - Table 3. TLD - PLATO differences indicate a mean deviation of $3.8 \%$. Since TLD data provides a scarce number of points relative far away from the most heterogeneous structures, a direct comparison between PLATO and GEANT4 dose matrices (one for each CT image used) was performed. Near air cavities in the head region, differences between GEANT4 and PLATO of $10 \%$ to $40 \%$ were found. Although a dose difference of $30 \%$ to $40 \%$ in air cavities itself have no clinical relevance, this very low density regions introduce a significant perturbation in electron and photon fluencies which are not accounted for in PLATO dose calculation algorithm. As a result PLATO dose values near air-tissue interfaces are $10 \%$ higher than those obtained with GEANT4. This differences can be seen, for example, in the dose line $3 \mathrm{~cm}$ away from beam central irradiation axis - Figure 7 and 12 a). Correspondent $\mathrm{CT}$ number distribution is shown in figure $12 \mathrm{~b}$ ). One should notice that since PLATO does not calculate dose outside the head contour, only the region between 10 to $25 \mathrm{~cm}$ can be compared with GEANT4. In tissue near air cavities GEANT4 dose values decrease down to $60-70 \%$. These results are consistent with measurements by other authors, who have found that near air cavities, like larynx, dose values calculated by conventional treatment planning systems could be overestimated by about $25 \%$ with opposed beam irradiations [24].

\footnotetext{
${ }^{6} n d f$ - number of degrees of freedom
} 
Deficits of this magnitude would be expected to have clinical consequences and need to be taken into account during treatment planning. The most probable effect of underdosage is to increase substantially the probability of tumor recurrence. In fact, for some early stages in larynx and naso-farynx underdosages between $5 \%$ to $18 \%$ are correlated with a decrease in tumor control probability from $75 \%$ to $50 \%$ [23]. Underdosage scenarios could be accurately resolved and identified by GEANT4 while the commercial treatment planning system was incapable of detecting it.

\section{Conclusions}

In this paper we have reported the implementation and validation of a dose calculation tool based on GEANT4 with photon beams in simple setups like homogeneous water phantoms and more complex anthropomorphic phantoms. GEANT4 simulation results agree with ionization chamber and TLD experimental data within the measurement and computational uncertainties. The comparison with PLATO have demonstrate the capability of GEANT4 in the benchmark of treatment planning systems dose calculation algorithms, specially in anatomical regions in which air cavities or low density volumes are present like the head and the lung. Due to the modular GEANT4 architecture thi calculation tool can be easily extended to perform dose calculations with other type of beams and radiation environments.

\section{Acknowledgments}

The authors would like acknowledge FCT - Fundação de Ciência e Tecnologia for financial support and Prof. Maria Grazia Pia (CERN/EP division and INFN-Genova) and Prof. Vladimir Ivanchenko (CERN/EP divison and Budker Institute for Nuclear Physics) from GEANT4 Collaboration for their assistance. 


\section{References}

[1] P. Andreo, Monte Carlo techniques in medical radiation physics, Phys. Med. Biol. 36 (7) (1991) 861-920.

[2] L. Wang, E. Yorke, C.-S. Chui, Monte Carlo evaluation of tissue inhomogeneity effects in the treatment of the head and neck, Int. J. Rad. Onc. Biol. Phys. 50 (5) (2001) 1339-1349.

[3] H. Zaidi, Relevance of accurate Monte Carlo modelling in nuclear medical imaging, Med. Phys. 26 (1999) 574-608.

[4] R. Taschereau, R. Roy, J. Pouliot, Relative biological effectiveness enhancement of a I-125 brachytherapy seed with characteristic $\mathrm{X}$ rays from its constitutive materials, Med. Phys. 29 (7) (2002) 1397-1402.

[5] M. Biaggi, F. Ballarini, W. Burkard, E. Egger, A. Ferrari, A. Ottolenghi, Physical and biophysical characteristics of a fully modulated $72 \mathrm{MeV}$ therapeutic proton beam: model predictions and experimental data, Nucl. Instr. and Meth. in Phys. Res. B 159 (1999) 89-100.

[6] S. Roesler, W. Heinrich, H. Schraube, Monte Carlo calculation of the radiation field at aircraft altitudes, Rad. Prot. Dosim. (2002) 367-388.

[7] E. R. Benton, E. V. Benton, Space radiation dosimetry in low-earth orbit and beyond, Nucl. Instr. and Meth. in Phys. Res. B 184 (2001) 255-294.

[8] M. R. Arnfield, C. Siantar-Hartmann, J. Sibers, P. Garmon, L. Cox, R. Mohan, The impact of electron transport on the accuracy of computed dose, Med. Phys. 27 (6) (2000) 1266-1274.

[9] R. D. Lewis, S. J. S. Ryde, A. W. Seaby, D. A. Hancock, C. J. Evans, Use of Monte Carlo computation in benchmarking radiotherapy treatment planning system algorithms, Phys. Med. Biol. 45 (2000) 1755-1764.

[10] ICRU Report 50, Prescribing, Recording and Reporting Photon Beam Therapy, Washington D.C., 1987.

[11] D. Miller (Ed.), AAPM Report 55 radiation treatment planning dosimetry verification, Radiation Therapy Committee Task Group \#23, American Institute of Physics, College Park, 1995.

[12] S Agostinelli et al GEANT4 Collaboration, GEANT4 - a simulation toolkit, Nucl. Instr. and Meth. in Phys. Res. A 506 (2003) 250-303.

[13] F. Brun, F. Rademakers, ROOT: An object-oriented data analysis framework, Linux J. (1998) 51.

[14] G. Barrand, P. Binko, M. Dönzelmann, A. Johnson, A. Pfeiffer, Abstract Interfaces for Data Analysis - Component Architecture for Data Analysis Tools, in: Proc. Int. Conf. Computing in High Energy and Nuclear Physics, September $3-7$, Beijing, P.R.China, 2001. 
[15] C. Manfredotti, U. Nastasi, R. Marchisio, C. Ongaro, G. Gervino, R. Ragona, S. Anglesio, G. Sannazzari, Monte Carlo simulation of dose distribution in electron beam radiotherapy treatment planning, Nucl. Instr. and Meth. in Phys. Res. A 291 (1990) 646-654.

[16] W. Schneider, T. Bortfeld, W. Schlegel, Correlation beteween CT numbers and tissue parameters needed for Monte Carlo simulations of clinical dose distributions, Phys. Med. Biol. 45 (2000) 459-478.

[17] A. Chaves, A. Alves, M. Fragoso, M. C. Lopes, C. Oliveira, L. Peralta, P. Rodrigues, J. Seco, A. Trindade, EGS4 and MCNP4b MC simulation of a Siemens KD2 accelerator in 6 MV photon mode, Tech. Rep. 01-01, LIP Preprint Note, http://weblib.cern.ch/abstract?EXT-2001-008 (2001).

[18] S. Chauvie, V. Ivanchenko, F. Longo, G. Santin, P. Nieminen, M. G. Pia, The Geant4 simulation toolkit and its low energy electromagnetic physics package, Med. Phys. 29 (6) (2002) 1231.

[19] C. Alves, A. Chaves, M. C. Lopes, C. Oliveira, L. Peralta, P. Rodrigues, A. Trindade, Buildup measurements and calculations for photon beams - search of the effective point of measurement for pinpoint and Markus chambers, Phys. Medica 17 (supl4) (2001) 23-25.

[20] U. Schneider, E. Pedroni, A. Lomax, The calibration of CT Hounsfield units for radiotherapy treatment planning, Phys. Med. Biol. 41 (1996) 111124 .

[21] T. Bortfeld, W. Schlegel, B. Rhein, Decomposition of pencil beam kernels for fast dose calculations in three-dimensional treatment planning, Med. Phys. 20 (2) (1993) 311-318.

[22] C. X. Yu, J. W. Wong, Implementation of the ETAR method for 3D inhomogeneity correction using FFT, Med. Phys. 20 (1993) 627-632.

[23] A. Wambersie, R. A. Gahbauer, Medical applications of electron linear accelerators, in: S. Turner (Ed.), Proceedings of CAS - The CERN Accelerator School: Cyclotrons, linacs and their applications, La Hulpe, Belgium, 28 April - 5 May, 1994, Vol. CERN 96-02, CERN, 1996.

[24] P. M. Ostwald, T. Kron, C. S. Hamilton, Assessment of mucosal underdosing in larynx irradiation, Int. J. Radiat. Oncol. Biol. Phys. 36 (1996) 181-187. 


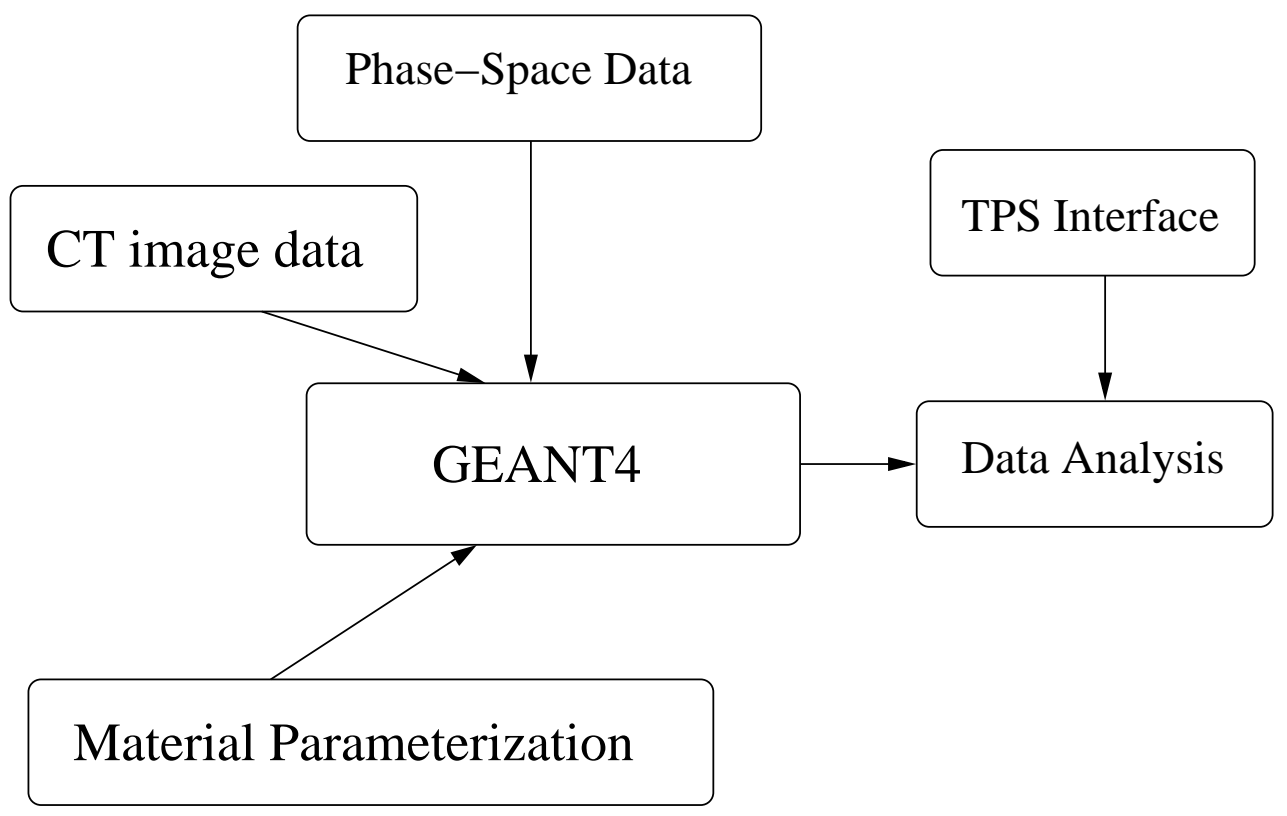

Figure 1: Overall organization of the developed GEANT4 dose calculation system. 


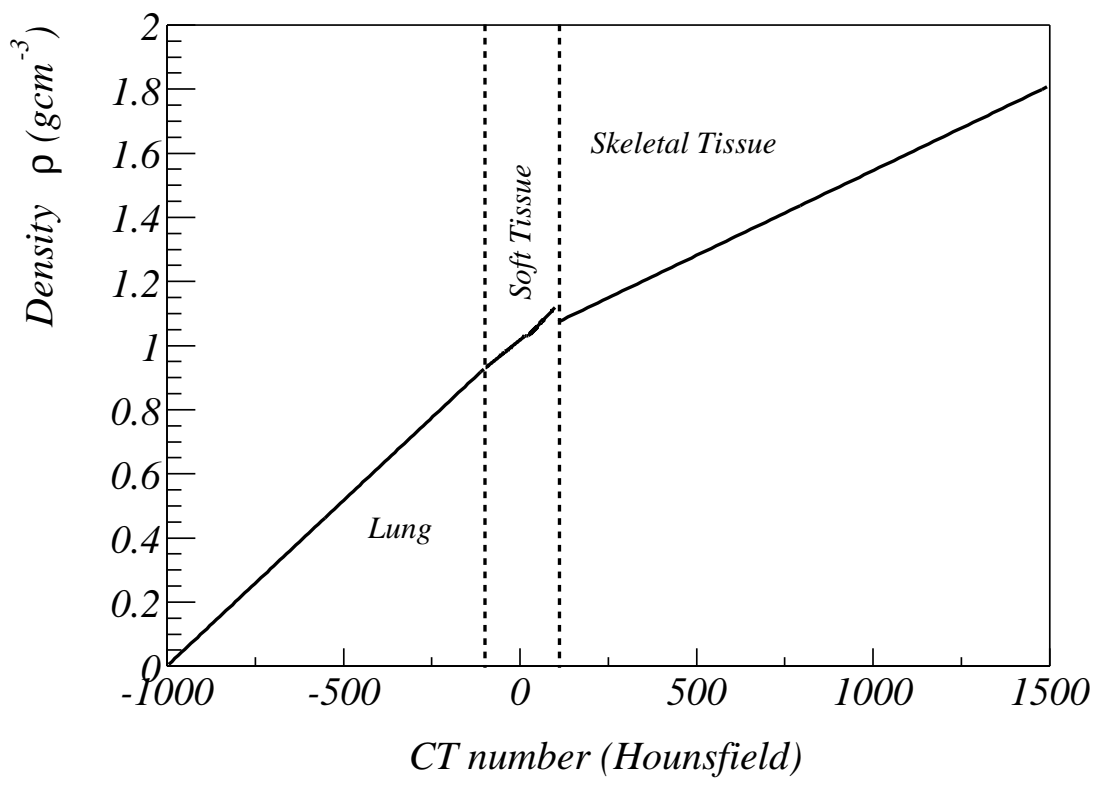

Figure 2: Implemented CT number to mass density parameterization.

Table 1: Calculated and tabulated density values for Alderson-Rando phantom lung and tissue.

\begin{tabular}{cccc}
\hline Material & $\begin{array}{c}\text { Mean CT number } \\
\text { Hounsfield }\end{array}$ & $\begin{array}{c}\rho \text { (GEANT4) } \\
\mathrm{gcm}^{-3}\end{array}$ & $\begin{array}{c}\rho \text { (Tabulated) } \\
\mathrm{gcm}^{-3}\end{array}$ \\
\hline Lung & $-671.2 \pm 15.8$ & $0.34 \pm 0.02$ & $0.32 \pm 0.01$ \\
Tissue & $+10.8 \pm 8.0$ & $1.01 \pm 0.02$ & $0.98 \pm 0.01$ \\
\hline
\end{tabular}




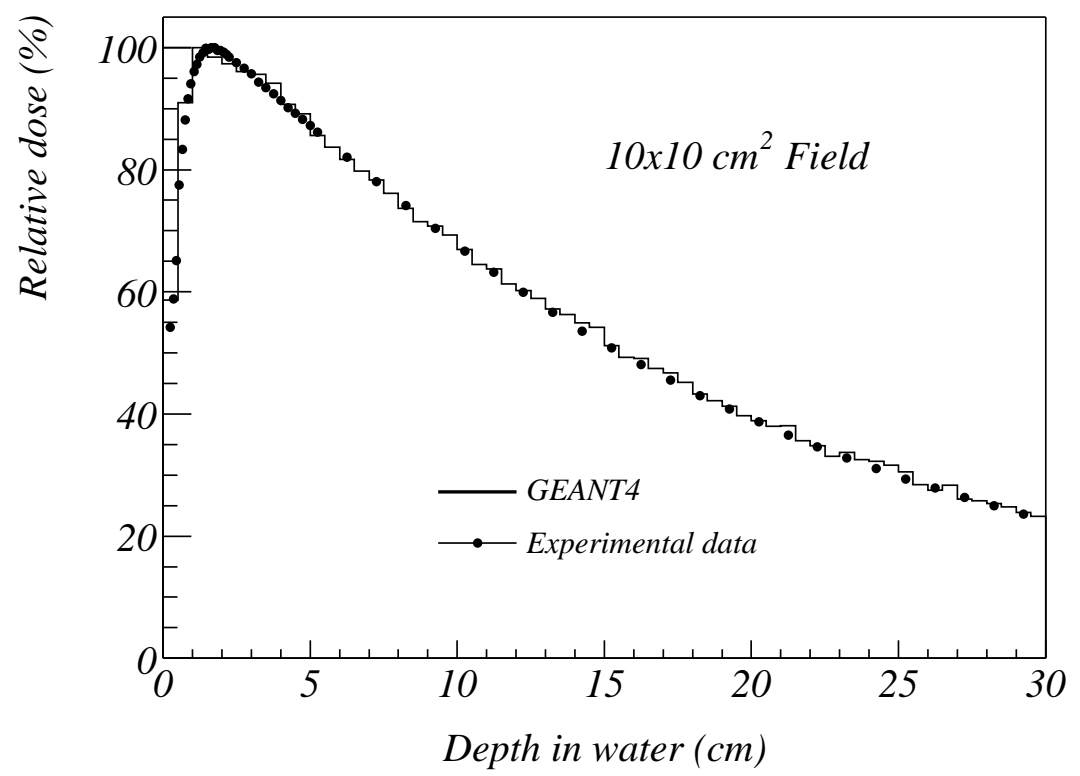

(a)

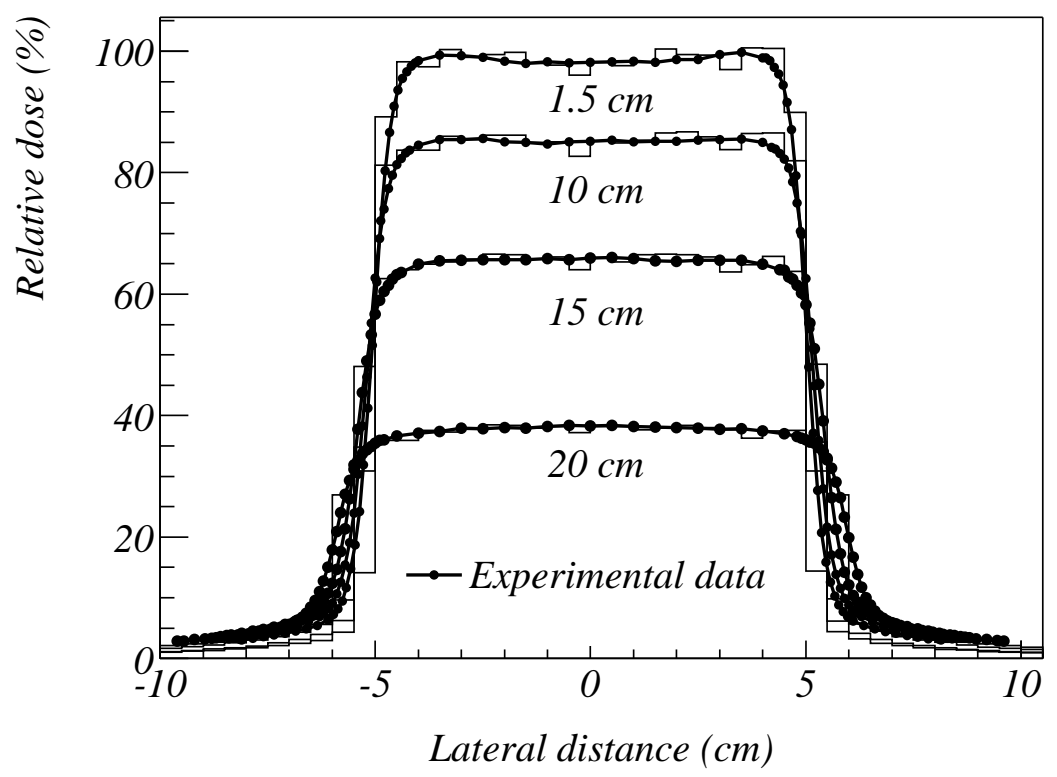

(b)

Figure 3: (a) Relative central axis depth dose curves and (b) transverse dose profile distributions at 1.5, 10, 15 and $20 \mathrm{~cm}$ depth in a water phantom for $10 \times 10 \mathrm{~cm}^{2} 6 \mathrm{MV}$ photon field. 


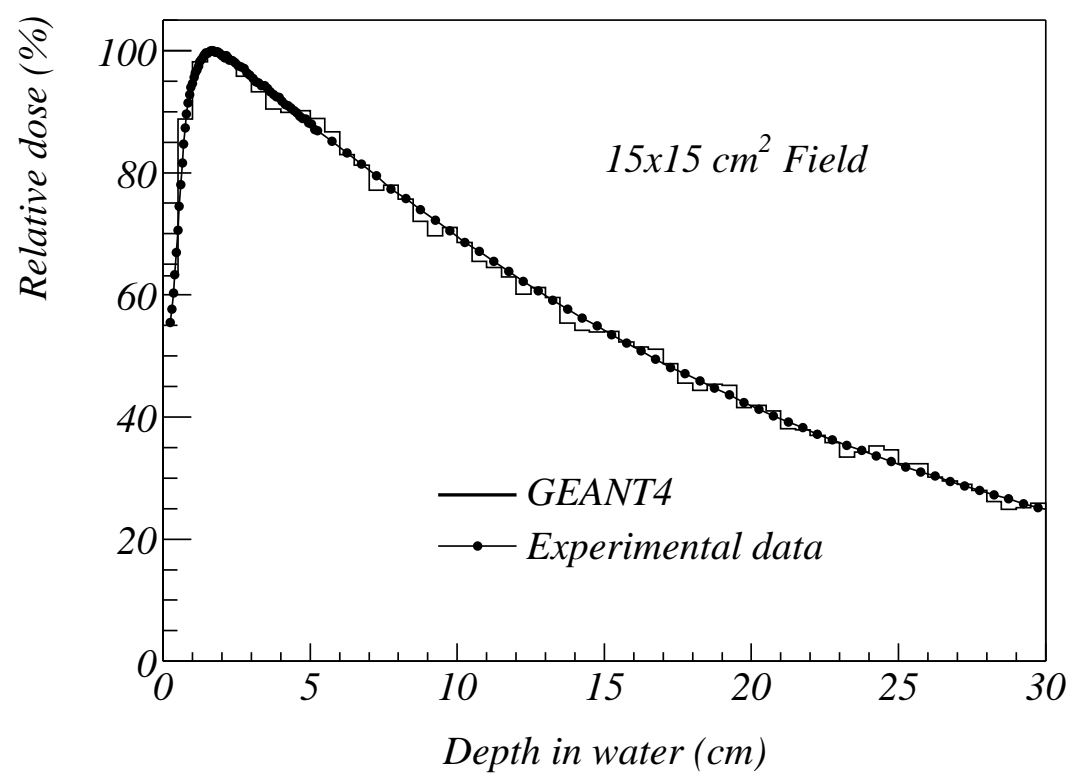

(a)

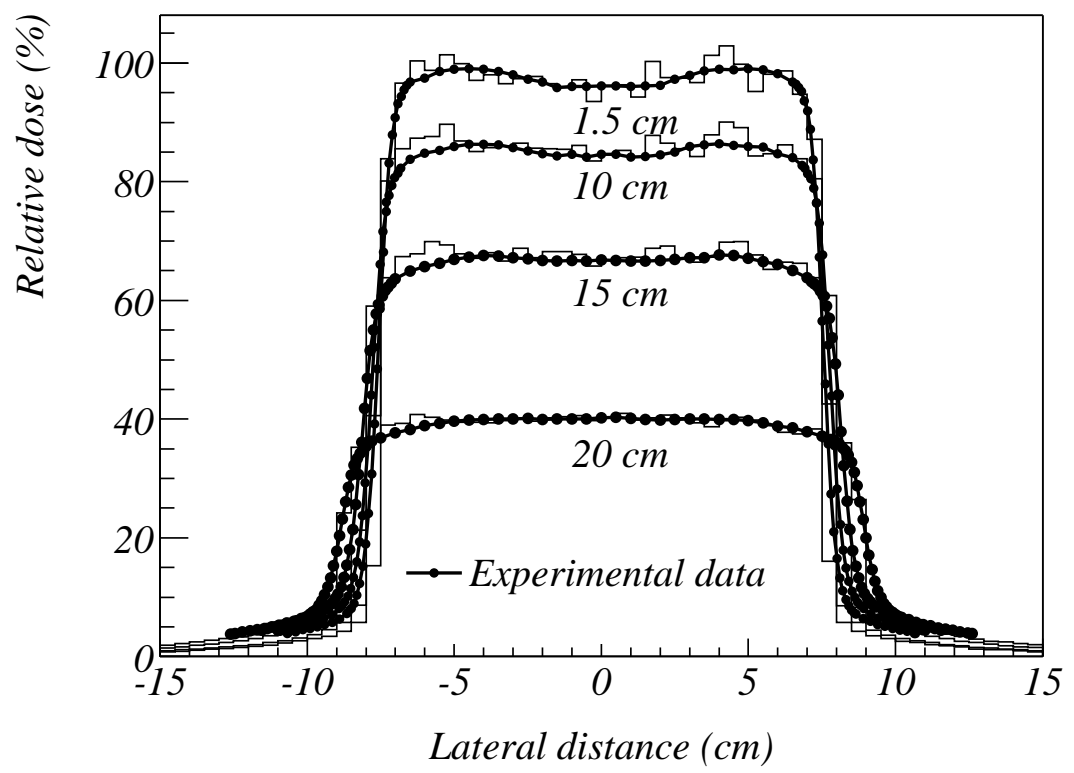

(b)

Figure 4: (a) Relative central axis depth dose curves and (b) transverse dose profile distributions at 1.5, 10, 15 and $20 \mathrm{~cm}$ depth in a water phantom for $15 \times 15 \mathrm{~cm}^{2} 6 \mathrm{MV}$ photon field. 


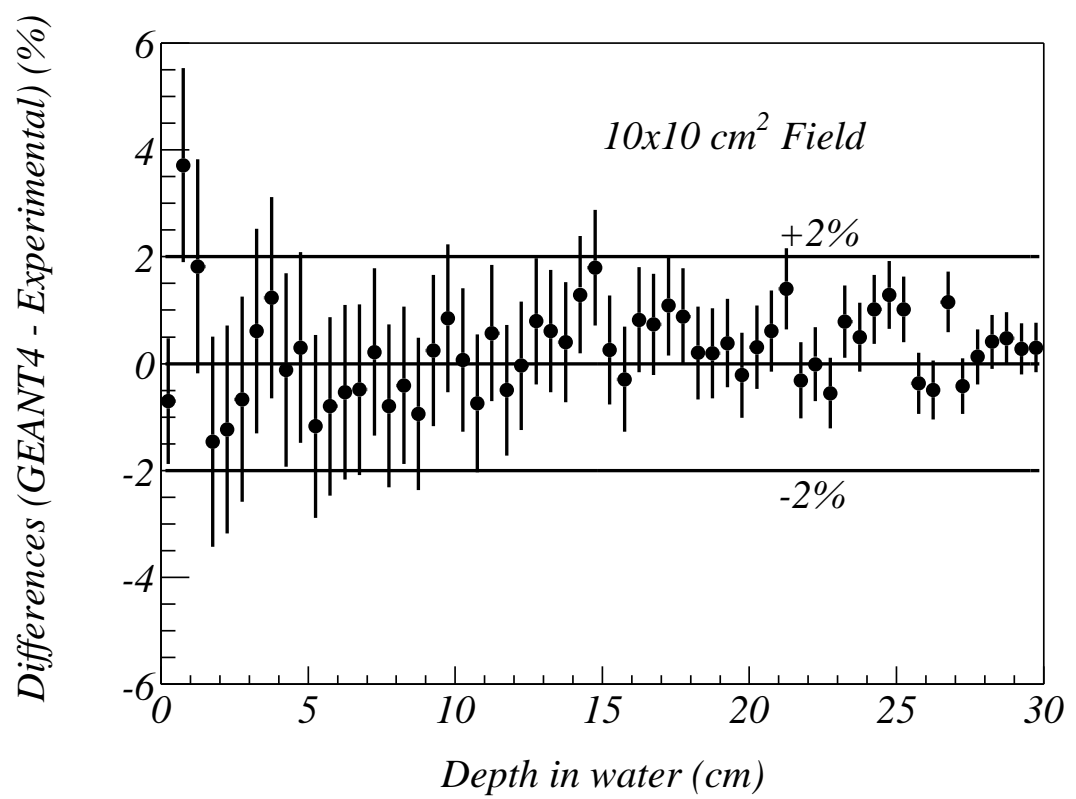

(a)

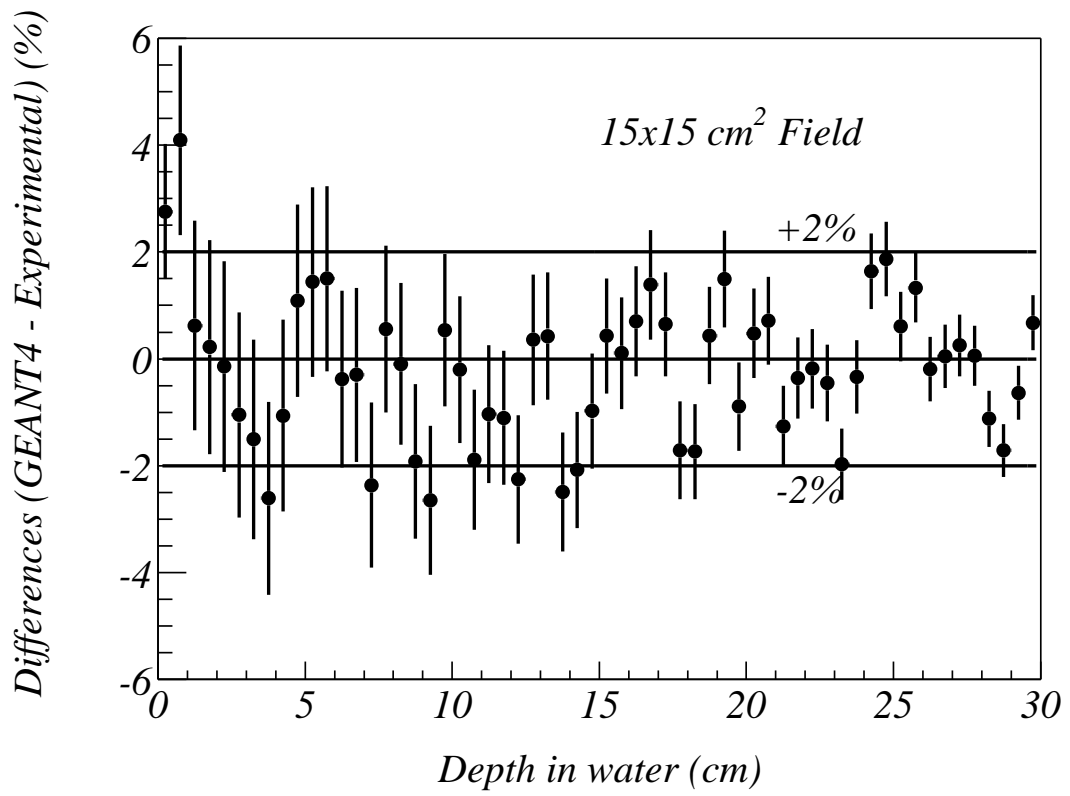

(b)

Figure 5: Differences in relative dose between GEANT4 and ionization chamber data in a water phantom for (a) $10 \times 10 \mathrm{~cm}^{2}$ and (b) $15 \times 15 \mathrm{~cm}^{2} 6 \mathrm{MV}$ photon field. 


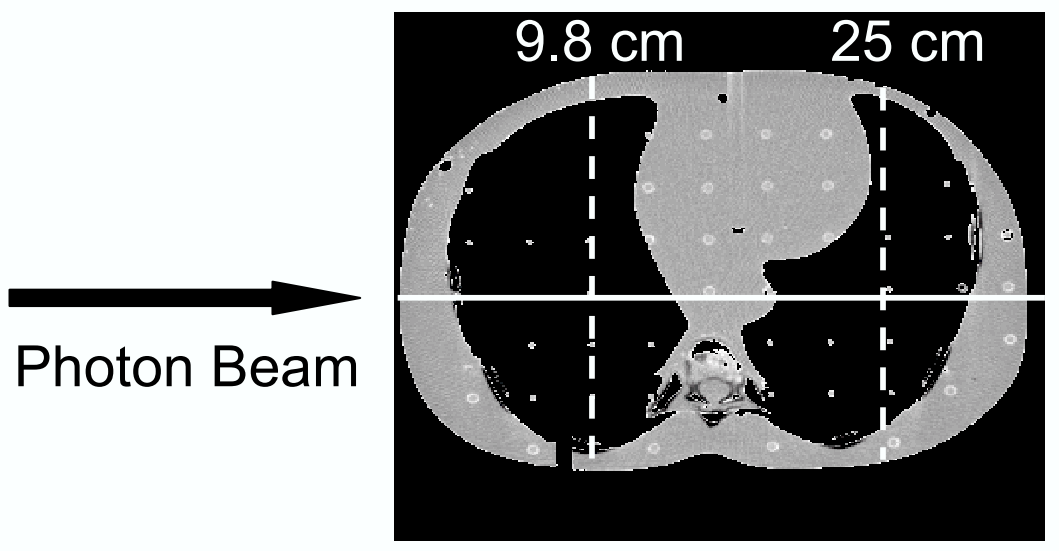

Figure 6: CT image for anthropomorphic thorax irradiation. Arrow indicates beam entry point and solid line indicate central axis of incidence. Profile dose distributions were obtained along perpendicular directions (dashed lines) to the beam axis at 9.8 and $25 \mathrm{~cm}$ from the beam entrance point.
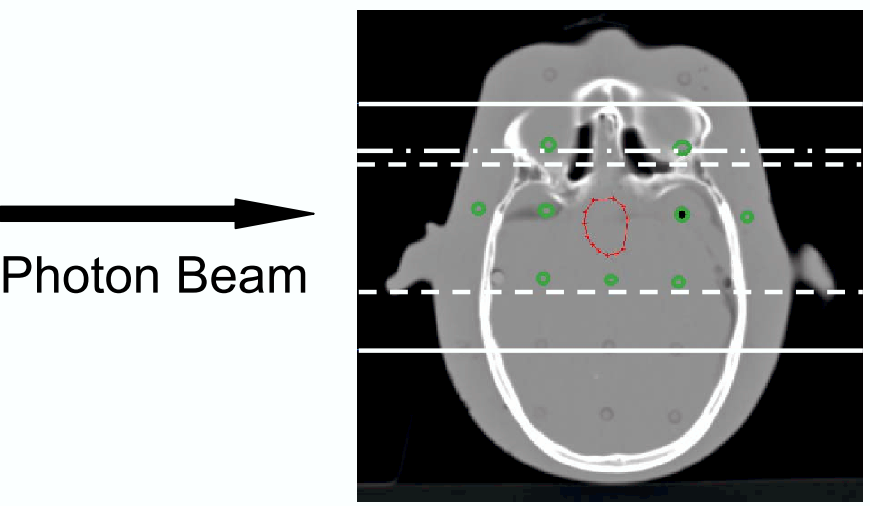

Photon Beam

Figure 7: Central CT image for anthropomorphic head irradiation. Solid lines and dashed lines indicate geometrical limits of $10 \times 10 \mathrm{~cm}^{2}$ and $5 \times 5 \mathrm{~cm}^{2}$ field, respectively. The thick dashed-point line represents a dose line located $3 \mathrm{~cm}$ away from beam central irradiation axis (see section 4.2)

Table 2: $\chi^{2}$, mean and maximum deviations for thorax irradiation (single field).

\begin{tabular}{llcc}
\hline System & $\chi^{2}$ & Mean Dev $(\%)$ & Max Dev $(\%)$ \\
\hline GEANT4 & 8.439 & 0.4 & 2.7 \\
PLATO & 107.343 & 4.0 & 10.7 \\
\hline ndf $=16$ & &
\end{tabular}




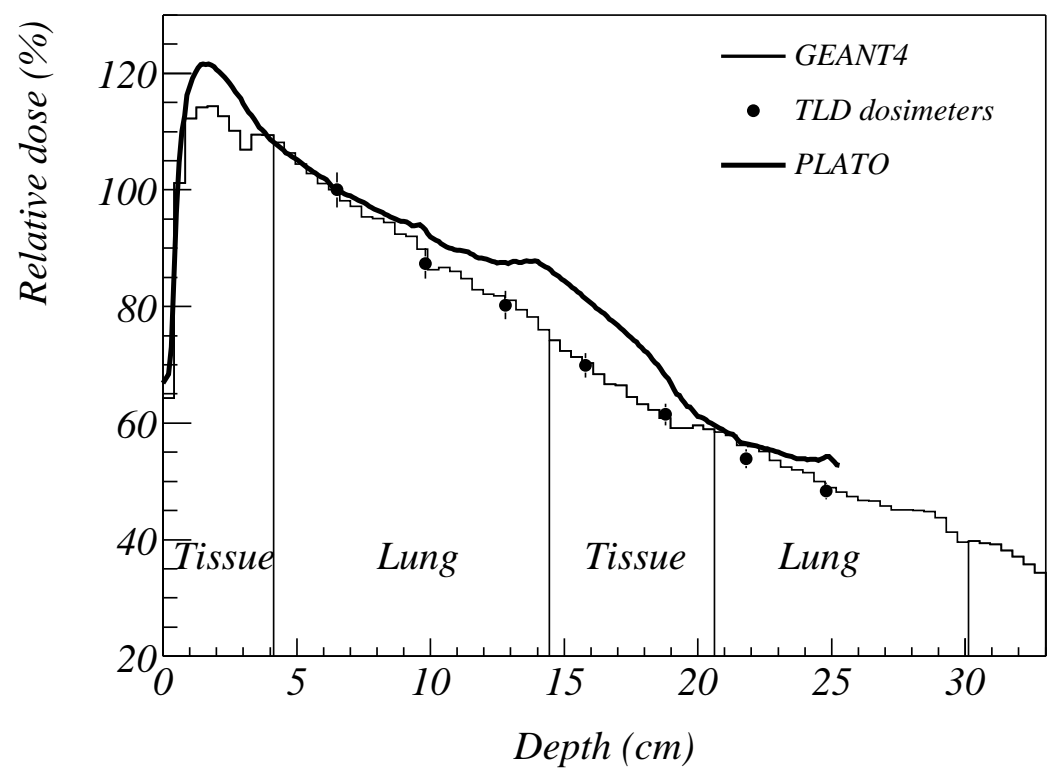

(a)

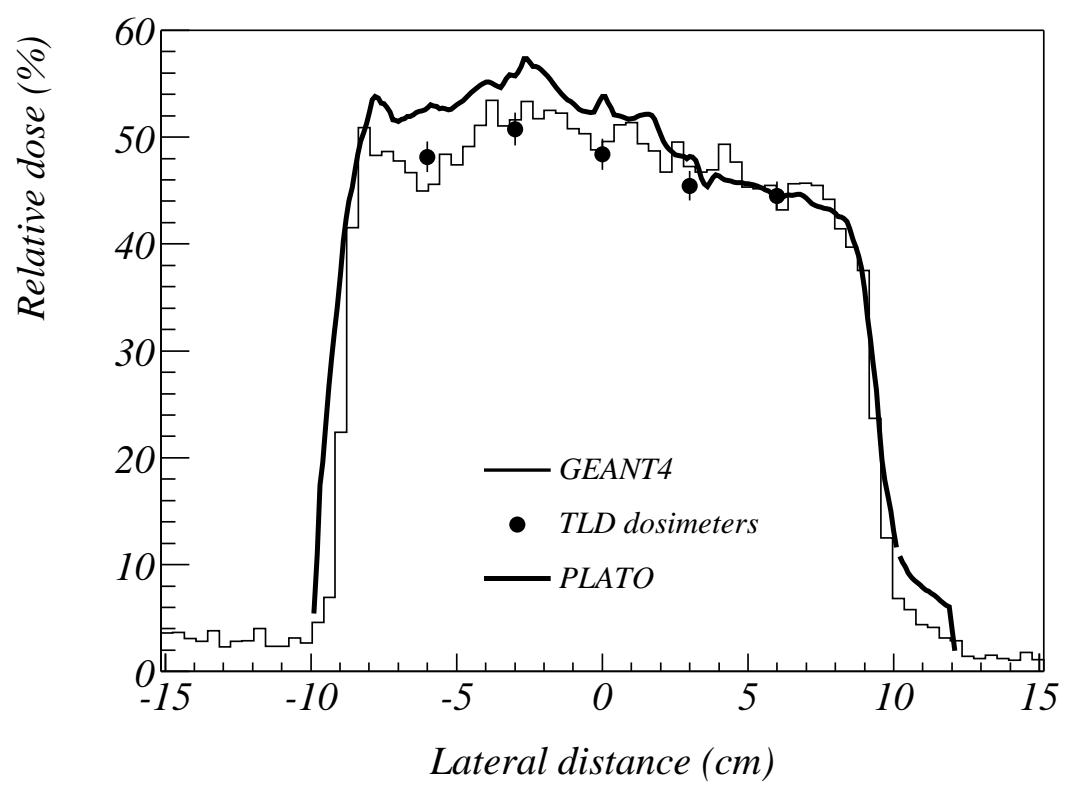

(b)

Figure 8: (a) Relative central axis depth dose curves and (b) transverse relative depth dose distributions at $25 \mathrm{~cm}$ depth for a $15 \times 15 \mathrm{~cm}^{2} 6 \mathrm{MV}$ single field Alderson-Rando thorax irradiation. 


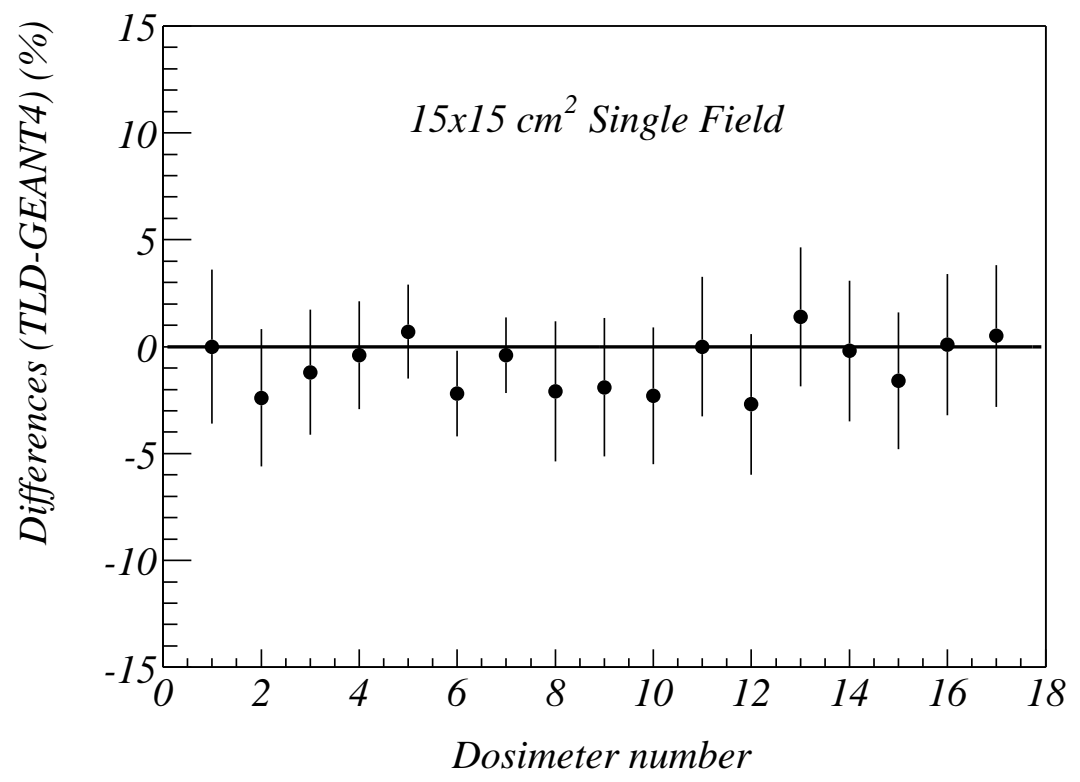

(a)

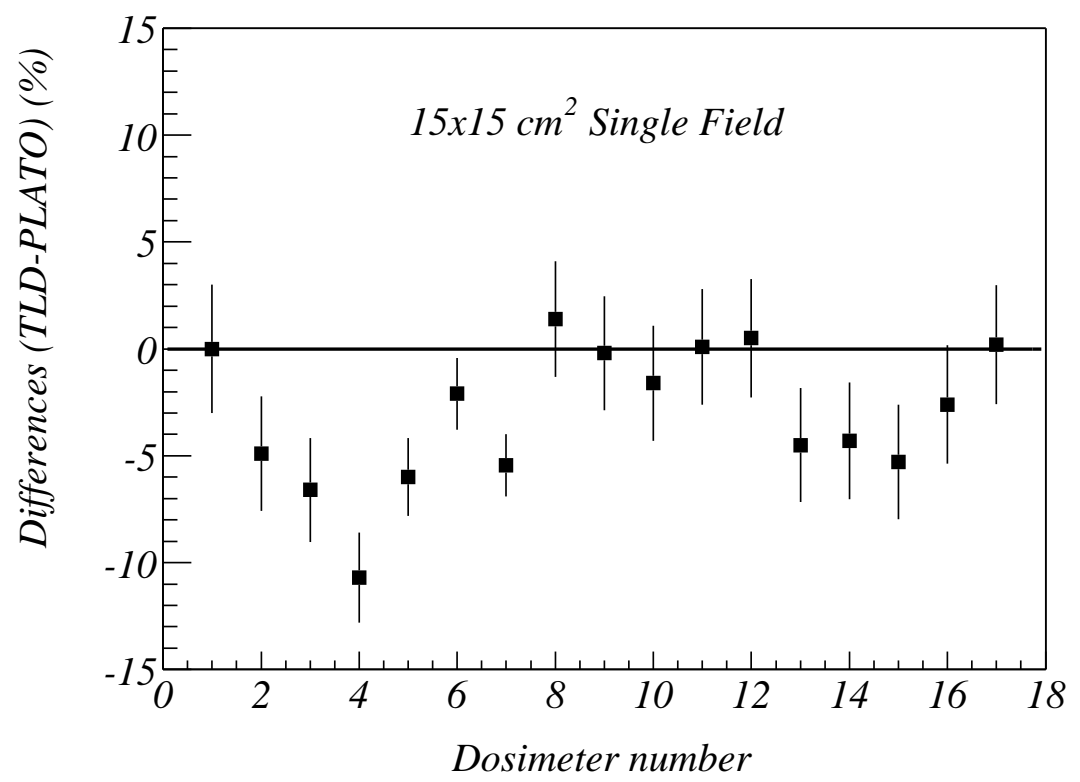

(b)

Figure 9: Differences in relative dose between (a) TLD and GEANT4 and (b) TLD and PLATO for a $15 \times 15 \mathrm{~cm}^{2} 6 \mathrm{MV}$ single field Alderson-Rando thorax irradiation. 


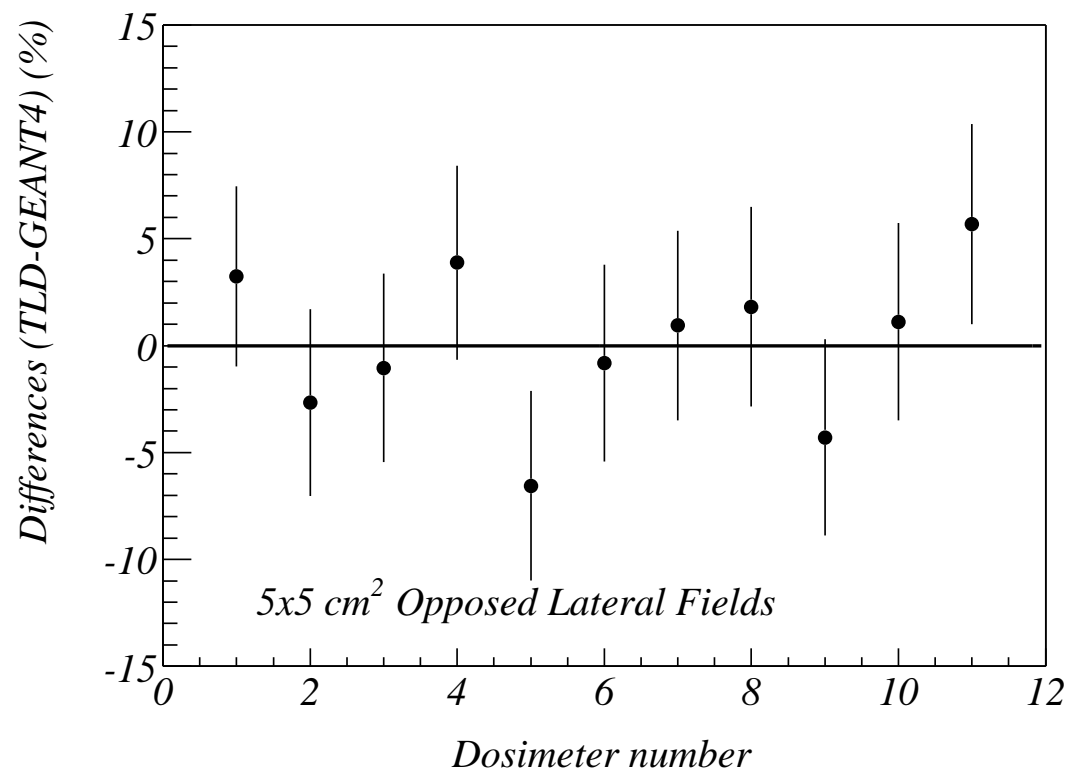

(a)

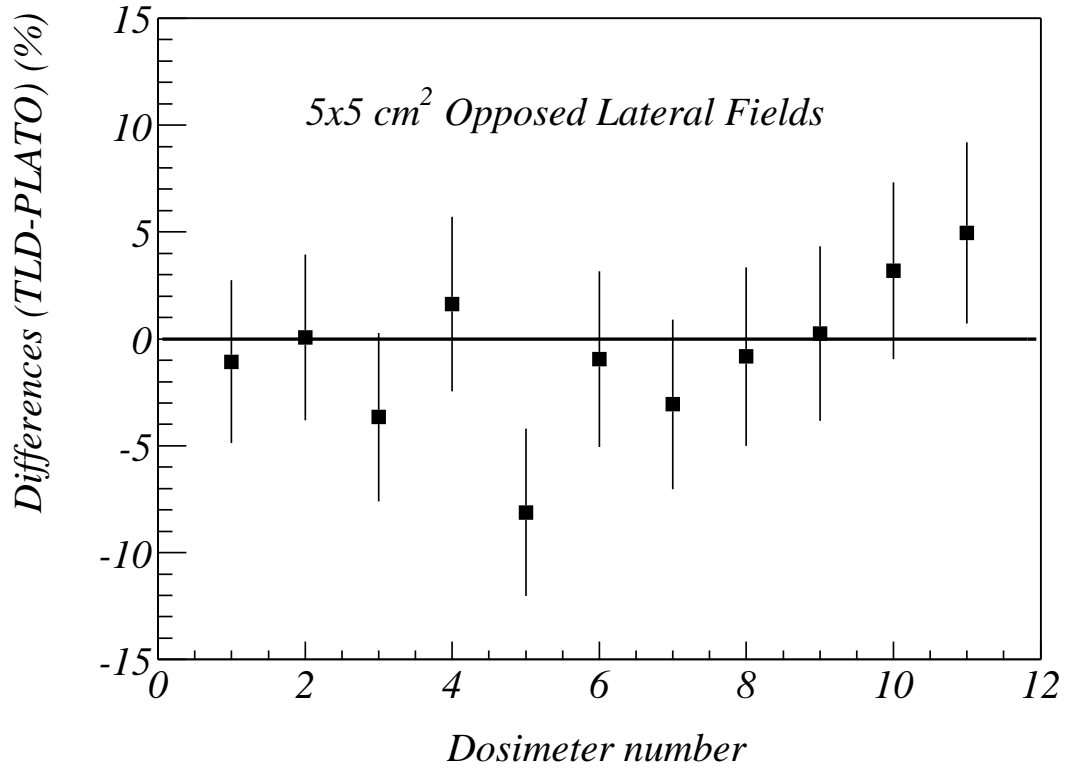

(b)

Figure 10: Differences in relative dose between (a) TLD and GEANT4 and (b) TLD and PLATO for a two opposed lateral $5 \times 5 \mathrm{~cm}^{2}$ fields Alderson-Rando head irradiation. 


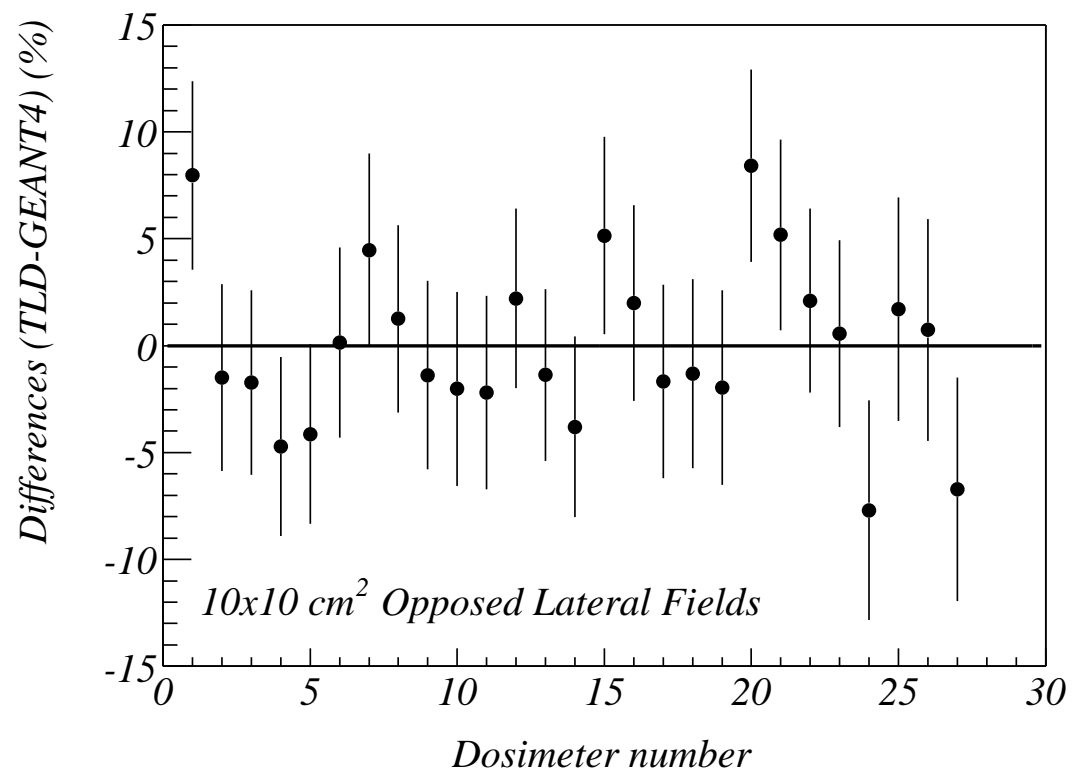

(a)

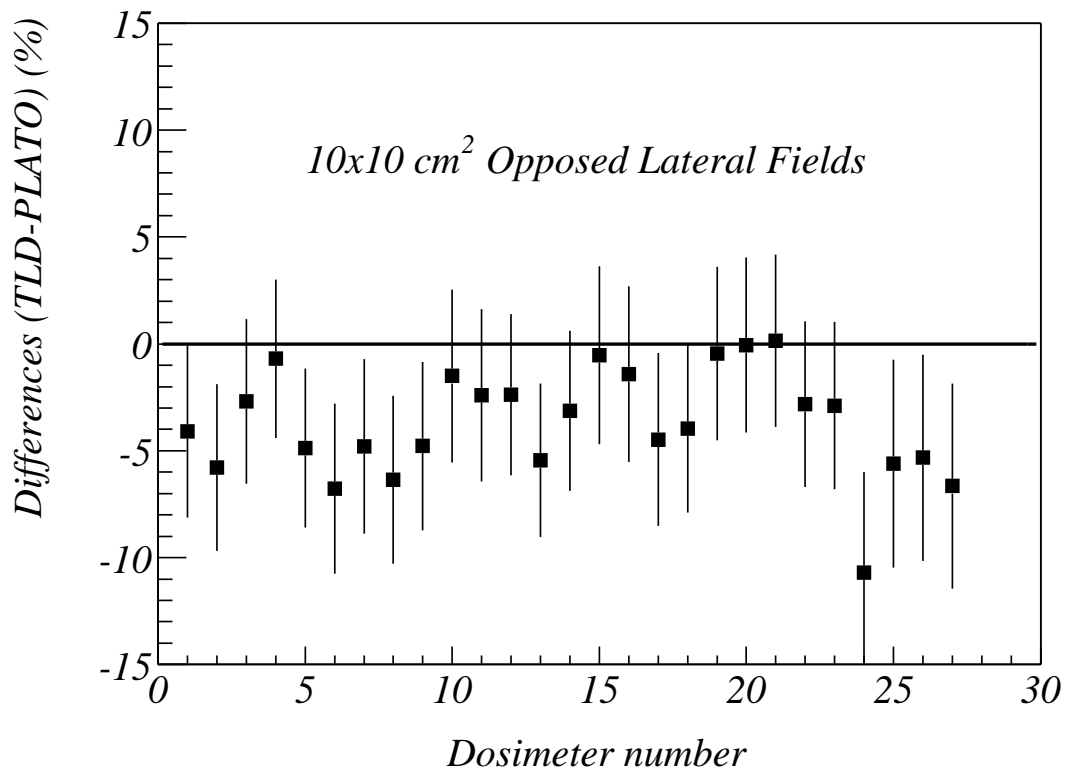

(b)

Figure 11: Differences in relative dose between (a) TLD and GEANT4 and (b) TLD and PLATO for a two opposed lateral $10 \times 10 \mathrm{~cm}^{2}$ fields Alderson-Rando head irradiation. 


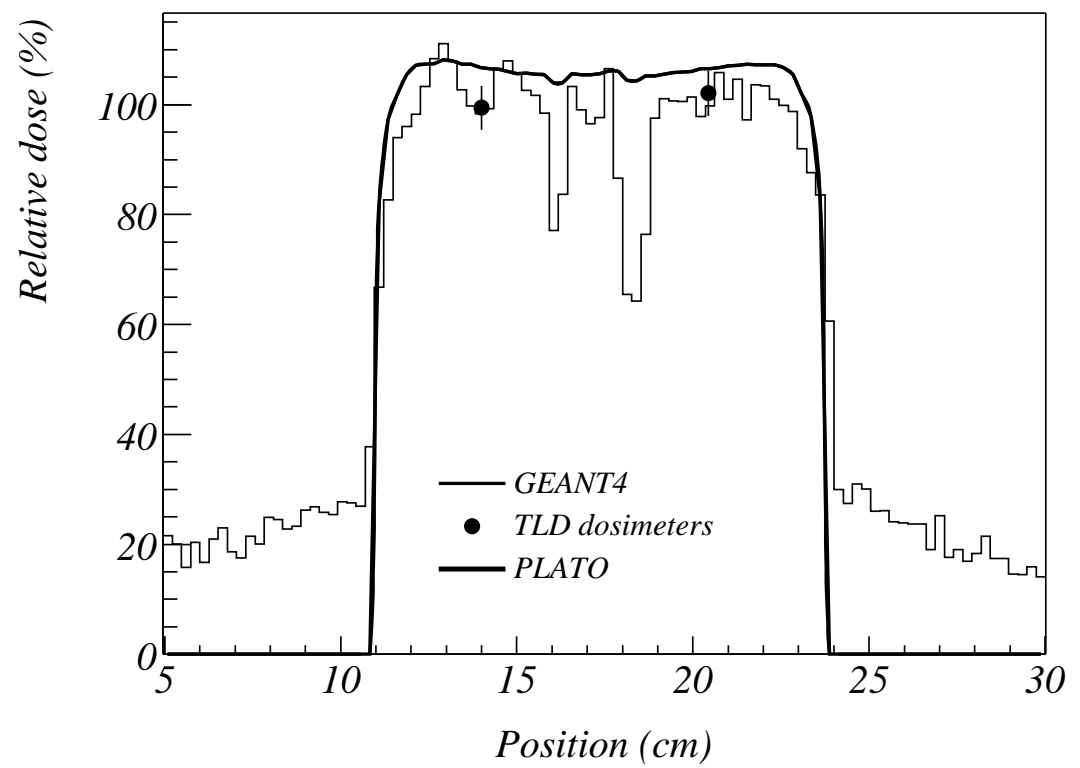

(a)

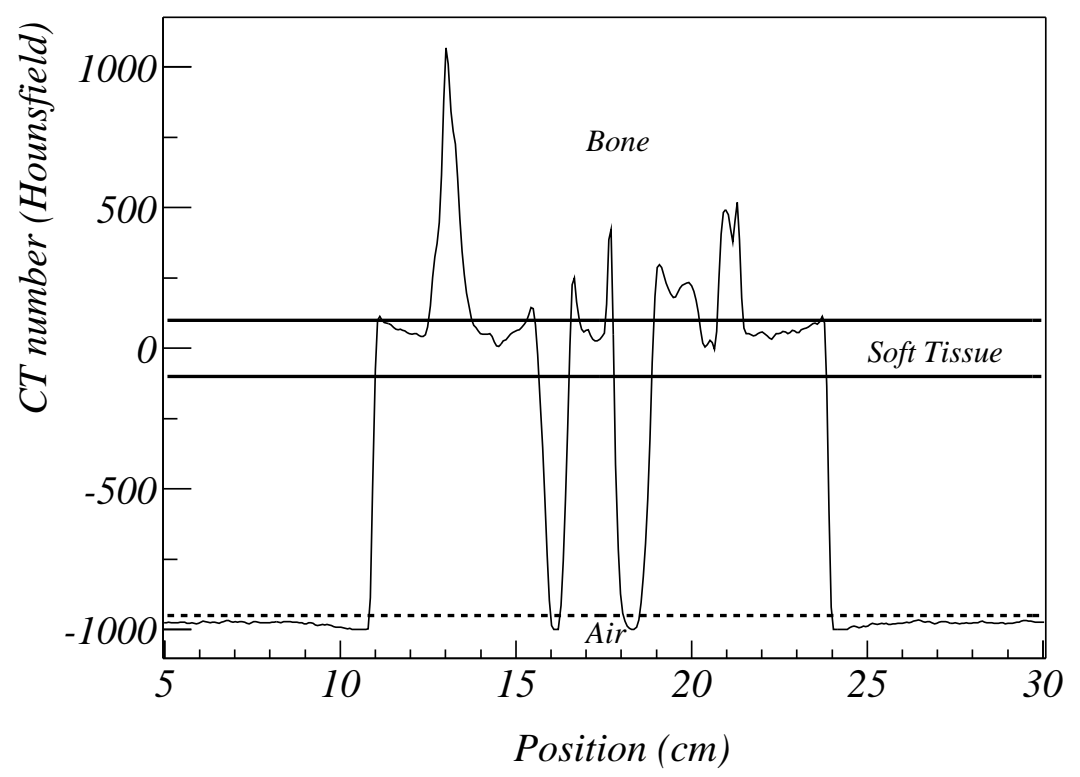

(b)

Figure 12: Depth dose distribution along $3 \mathrm{~cm}$ above beam central axis for head irradiation (a) and correspondent CT number distribution in function of position (b). 
Table 3: $\chi^{2}$, mean and maximum deviations for head irradiation (opposed lateral fields).

\begin{tabular}{lllcc}
\hline Field & System & $\chi^{2}$ & Mean Dev (\%) & Max Dev (\%) \\
\hline $5 \times 5 \mathrm{~cm}^{2, \mathrm{a}}$ & GEANT4 & 8.046 & $0.1 \pm 2.04$ & 6.6 \\
& PLATO & 8.255 & $0.7 \pm 2.04$ & 8.1 \\
\hline $10 \times 10 \mathrm{~cm}^{2, \mathrm{~b}}$ & GEANT4 & 24.271 & $0.0 \pm 5.9$ & 8.4 \\
& PLATO & 43.918 & $3.8 \pm 4.0$ & 10.7 .4 \\
\hline
\end{tabular}

${ }^{\mathrm{a}}$ ndf $=10,{ }^{\mathrm{b}}$ ndf $=26$ 